\title{
Intrahepatic Cholangiocarcinoma Cells Promote Epithelial-mesenchymal Transition of Hepatocellular Carcinoma Cells by Secreting LAMC2
}

Wenda Cen, $\mathrm{MS}^{*}$; Jiandong Li, MS2,3*; Chenhao Tong, MS²,3; Weiguang Zhang, PhD"; Yunfeng Zhao, PhD5; Baochun Lu, MD 2,3 ; Jianhua Yu, MD2,3凶

1. Shaoxing University School of Medicine, Shaoxing, China.

2. Department of Hepatobiliary Surgery, The First Affiliated Hospital of Shaoxing University, Shaoxing, China.

3. Department of Hepatobiliary Surgery, Shaoxing People's Hospital (Shaoxing Hospital, Zhejiang University School of Medicine), Shaoxing, China.

4. Department of Molecular Medicine and Clinical Laboratory, Shaoxing Second Hospital, Shaoxing, China.

5. Department of Pharmacology, Toxicology \& Neurosciences, LSU Health Sciences Center, Shreveport, LA, USA.

* These authors contributed equally to this work.

$\square$ Corresponding author: Jianhua Yu: Department of Hepatobiliary Surgery, The First Affiliated Hospital of Shaoxing University, Shaoxing, China; Department of Hepatobiliary Surgery, Shaoxing People's Hospital (Shaoxing Hospital, Zhejiang University School of Medicine); 568 North Zhongxing Road, Shaoxing, 312000, China. E-mail: yujianhua@zju.edu.cn. Phone numbers: +86 15157552933. Phone: 86-575-8822 9383. Fax: 86-575-8513 8402.

(C) The author(s). This is an open access article distributed under the terms of the Creative Commons Attribution License (https://creativecommons.org/licenses/by/4.0/). See http://ivyspring.com/terms for full terms and conditions.

Received: 2020.11.08; Accepted: 2021.03.19; Published: 2021.04.19

\begin{abstract}
Hepatocellular carcinoma and intrahepatic cholangiocarcinoma cells are common primary hepatic tumor cells in the liver. Combined hepatocellular cholangiocarcinoma $(\mathrm{CHC})$ contains both hepatocellular carcinoma cells and intrahepatic cholangiocarcinoma cells in one tumor lesion and these tumors show poor prognosis. Here we examined the potential interaction between hepatocellular carcinoma cells and intrahepatic cholangiocarcinoma cells using cell culture studies. The results showed that culture supernatant from intrahepatic cholangiocarcinoma cells induced endothelial-mesenchymal transition and facilitated the migration and invasion of hepatocellular carcinoma cells, although it did not accelerate the proliferation of hepatocellular carcinoma cells. Furthermore, culture supernatant from intrahepatic cholangiocarcinoma cells increased the chemoresistance of hepatocellular carcinoma cells. Laminin subunit gamma 2 (LAMC2) was detected in the culture supernatant of intrahepatic cholangiocarcinoma cells but not in that of hepatocellular carcinoma cells. Using established LAMC2 knockout intrahepatic cholangiocarcinoma cells, our results demonstrated that intrahepatic cholangiocarcinoma cells promoted the epithelial-mesenchymal transition of hepatocellular carcinoma cells through secreting LAMC2. Our results have revealed a novel mechanism of interaction between intrahepatic cholangiocarcinoma cells and hepatocellular carcinoma cells, which may provide new insight into developing effective treatments for $\mathrm{CHC}$.
\end{abstract}

Key words: Hepatocellular carcinoma; Intrahepatic Cholangiocarcinoma; Combined hepatocellular cholangiocarcinoma; LAMC2; EMT.

\section{Introduction}

Hepatocellular carcinoma (HCC) is the most common type of primary liver cancer, accounting for approximately $80 \%$ of all cases, and intrahepatic cholangiocarcinoma (ICC) is the second most common liver cancer, accounting for about $15 \%$ of cases [1]. HCC is comprised of hepatocellular carcinoma cells (HCC cells) and ICC is comprised of intrahepatic cholangiocarcinoma cells (ICC cells). Besides, there is a form of primary liver carcinoma contains a mixture of HCC cells and ICC cells, namely combined hepatocellular cholangiocarcinoma ( $\mathrm{CHC})$, with an incidence varying from $1.0 \%$ to $14.3 \%$ of primary liver cancer in different studies [1-5]. Although the number of CHC cases is relatively small, 
it is difficult to diagnose before surgery and has a dismal prognosis $[1,6]$. The data from a long-term prognosis study of liver cancer indicates that $\mathrm{CHC}$ has a significantly worse prognosis than HCC and ICC even after curative resection [1]. Given the special histological characteristics of $\mathrm{CHC}$, we speculated that the unique cellular component of $\mathrm{CHC}$ could contribute to its dismal prognosis.

Epithelial-mesenchymal transition (EMT), a critical process in which cancer cells gain enhanced invasion, migration, and metastasis abilities, has been associated with poor prognosis [7]. Cells undergoing EMT show loose or no interactions among cells, lost the expression of certain adhesion molecules such as E-cadherin, and acquire the expression of mesenchymal-associated markers such as vimentin and snail $[7,8]$. It has been shown that EMT is an important contributing factor to the development and progression of liver cancer $[9,10]$.

Laminins, a large family of extracellular matrix proteins, are comprised of three different chains $(\alpha, \beta$ and $\gamma$ ). Laminin-332 is a well-studied member of laminins and has been reported as an important regulator in the process of oncogenesis [11, 12]. $L A M C 2$ encodes the $\gamma 2$ chain of laminin-332 and is an important EMT-associated gene in various cancers including bladder cancer, colorectal cancer, lung cancer, and cholangiocarcinoma [13-16]. Early studies have reported that LAMC2 exists in the tumor microenvironments and influences the invasion and migration of cancer cells $[17,18]$.The tumor microenvironment plays a critical role in influencing the biological behavior of cancer cells. We speculate that there might be interactions between the HCC and ICC components in $\mathrm{CHC}$, which contribute to the poor prognosis of $\mathrm{CHC}$. For this purpose, we incubated HCC cells with culture supernatant from ICC cells, and examined possible mechanisms of action.

\section{Material and Methods}

\section{Reagents}

Cisplatin and doxorubicin were purchased from Cayman Chemical (Ann Arbor, MI, USA). Antibodies against $\beta$-actin (sc-47778), LAMC2 (sc-28330), E-cadherin (sc-71009), Vimentin (sc-80975) and Snail (sc-271977) were purchased from Santa Cruz Biotechnology, Inc. (Dallas, TX, USA).

\section{Cell culture}

The HepG2 and SNU-449 cell lines were obtained from the American Type Culture Collection (Manassas, VA, USA). The HCCC-9810 cell line and PLC/PRF/ 5 cell line were obtained from the Chinese Academy of Sciences Shanghai Branch Cell Bank
(Shanghai, China). The HuCCT1 cell line was purchased from the RIKEN BioResource Center (Ibaraki, Japan). HepG2, SNU-449 and PLC/PRF/5 cell lines are hepatocellular carcinoma cells, whereas HCCC-9810 and HuCCT1 cell lines are intrahepatic cholangiocarcinoma cells. HepG2, SNU-449, PLC/PRF/5, HCCC-9810 and HuCCT1 cells were cultured in RPMI 1640 medium (Gibco, Thermo Fisher Scientific, Inc.) supplemented with 10\% fetal bovine serum (FBS, Gibco, Thermo Fisher Scientific, Inc.), $100 \mathrm{IU} / \mathrm{ml}$ penicillin and $100 \mu \mathrm{g} / \mathrm{ml}$ streptomycin. After informed consent was obtained from patients and the tissue acquisition protocol was approved by the Ethics Committee of Shaoxing People's Hospital, liver explant tissue from a female patient with hepatic hemangioma without other hepatic diseases was obtained. Human cholangiocytes were isolated from normal liver tissue samples by magnetic bead-purification (Miltenyi Biotec, Inc.), and were cultured with DMEM/F12 medium (Gibco, Thermo Fisher Scientific, Inc.) as previously described [19].

\section{Preparation of cell culture supernatants}

Cells were plated in a $100-\mathrm{mm}$ dish at $40 \%-50 \%$ confluence, and the culture medium was replaced with fresh RPMI 1640 medium after cells attached to the plate. Twenty-four hours later, culture supernatant was collected and filtrated through a 0.22 $\mu \mathrm{m}$ filter (Millipore, Merck KGaA, MA, USA). The filtrated culture supernatants from HepG2, SNU-449, PLC/PRF/5, HCCC-9810, HuCCT1 cells, and cholangiocytes were used for subsequent experiments.

\section{Colony formation assay}

HepG2, PLC/PRF/5 and SNU-449 cells were seeded in a 12-well plate at 100 cells per well. After cells being attached, the culture medium was replaced with filtrated culture supernatants from either the same cell line (e.g. HepG2 clones were cultured with culture supernatants from HepG2 cells), cholangiocarcinoma cell lines, or cholangiocytes.

The culture supernatants were replaced every 3 days. After 14 days, cells were fixed in $4 \%$ paraformaldehyde for $15 \mathrm{~min}$. After washing, cells were stained with $0.005 \%$ crystal violet solution for 1 $\mathrm{h}$. The plates were aspirated, washed, and allowed to air dry. The morphology of cell colonies was observed using light or fluorescence microscopy.

\section{Western blot analysis}

Protein samples (20 $\mu \mathrm{g})$ of whole-cell lysate were separated on a $10 \%$ SDS-PAGE gel and transferred onto a polyvinylidene fluoride (PVDF) membrane. The membrane was blocked and incubated with a 
primary antibody, followed by incubation with a horseradish peroxidase-conjugated secondary antibody (Jackson ImmunoResearch Inc., West Grove, PA, USA). Immunoreactive bands were visualized using a chemiluminescence solution (Genesee Scientific, El Cajon, CA, USA). Experiments were repeated three times. $\beta$-actin was used as the loading control for cell lysis. The protein bands were quantified using the Image software.

For analysis of culture supernatants, the culture medium was centrifuged and filtrated with a $0.22 \mu \mathrm{m}$ filter to remove cells. Supernatant samples $(30 \mu \mathrm{l})$ were combined with $4 \times$ loading buffer $(10 \mu \mathrm{l})$, denatured, separated by SDS-PAGE, and analyzed as described above. Ponceau S staining of the PVDF membrane was used as the loading control for electrophoresis of the culture medium.

\section{Wound healing assays}

HepG2 and SNU-449 cells $\left(2 \times 10^{4}\right)$ were seeded in 96-well plates (Essen BioScience, Ann Arbor, MI, USA) and cultured overnight. Scratches were introduced by a Woundmaker (Essen BioScience). Wells were washed twice with serum-free medium, and different culture supernatants were added. After $48 \mathrm{~h}$, images were obtained and analyzed. Six replicate wells from each cell treatment were analyzed.

\section{Transwell invasion assays}

After culture overnight in serum-free medium, cells $\left(1 \times 10^{4}\right)$ in $0.1 \mathrm{ml}$ serum-free culture supernatant were placed into the upper chamber of a transwell insert (Corning, NY, USA) coated with Matrigel (BD Biosciences, San Jose, CA, USA). $0.5 \mathrm{ml}$ culture supernatant with $10 \%$ FBS was added to the lower chamber. Twenty-four hours later, cells that migrated to the other side of the insert were fixed with $4 \%$ paraformaldehyde for $15 \mathrm{~min}$ following by crystal violet staining. These experiments were repeated three times.

\section{Cell proliferation assays}

Equal numbers of cells were seeded in 96-well plates and incubated with different culture supernatants. Cell growth was determined using the 3-[4,5-dimethylthiazol-2-yl]-2, 5-diphenyltetrazolium bromide assay (MTT) assay (Sigma, St. Louis, MO, USA). One-tenth volume of MTT diluted in serum-free medium was added to each well at different time points, and the plates were incubated at $37^{\circ} \mathrm{C}$ for $4 \mathrm{~h}$. Formazan crystals were dissolved in DMSO, and the absorbance at $595 \mathrm{~nm}$ was measured using a spectrometer (Bio-Rad, Philadelphia, PA, USA). Six replicate wells from each cell type were analyzed.

\section{EdU proliferation assays}

HepG2 cells $\left(2 \times 10^{3}\right)$ were seeded into 6-well plates and allowed to attach overnight. The culture medium was replaced with different culture supernatants containing $10 \mu \mathrm{M}$ EdU (Beyotime, Nanjing, China) for $2 \mathrm{~h}$. Cells were washed with PBS and fixed with cold $4 \%$ paraformaldehyde. After treating with $0.3 \%$ TritonX-100, washing with PBS and blocking, cells were incubated with Azide 555 and Hoechst 33342 (Beyotime). Fluorescence was immediately detected using a Nikon microscope (Tokyo, Japan). Experiments were repeated three times.

\section{Drug sensitivity assays}

Cisplatin and doxorubicin were used to evaluate the effect of different culture supernatants on the chemotherapy sensitivity of the cells. Cells $\left(4 \times 10^{3}\right)$ were seeded into 96-well plates and allowed to attach overnight. Cells were then treated with the different culture supernatants containing various concentrations of cisplatin and doxorubicin. Twenty-four hours later, viable cells were determined using the MTT assay as described above.

\section{Cell apoptosis analysis}

HepG2 cells were treated with different culture supernatants for $24 \mathrm{~h}$. Cisplatin was added to culture supernatants at a final concentration of $1 \mu \mathrm{g} / \mathrm{ml}$. Four hours later, cells were collected and stained with Annexin V-FITC and propidium iodide (PI) (Becton-Dickinson, Franklin, NJ, USA) following the manufacturer's instructions. Apoptotic cells were quantified using a flow cytometer (Beckman Coulter, Fullerton, CA, USA). Experiments were repeated three times.

\section{Caspase 3 activity detection}

Caspase 3 activity in HepG2 cells was detected using the Caspase 3 Activity Assay Kit (Beyotime) following the manufacturer's protocol. The absorbance was measured at $405 \mathrm{~nm}$, and the relative activity of Caspase 3 was calculated.

\section{Establishment of LAMC2 stable knockout (KO) clones}

HuCCT1 cells were transfected with the Laminin $\mathrm{Y}^{-2}$ CRISPR/Cas9 KO Plasmid (Santa Cruz Biotechnology), which consists of a pool of three plasmids, encoding the Cas9 nuclease and a target-specific $20 \mathrm{nt}$ guide RNA. At $72 \mathrm{~h}$ post-transfection, cells were sorted using flow cytometry by expressing green fluorescence (GFP). The selected clones were amplified and Western blot analysis was performed to select LAMC2 KO clones. 


\section{Clinical tissue samples}

Tissue samples were obtained from patients with liver tumors who had a definite pathological diagnosis after surgery at the Shaoxing People's Hospital from January 2013 to February 2018. Informed consent was obtained from patients and the tissue acquisition protocol was approved by the Ethics Committee of Shaoxing People's Hospital. Definite HCC components and ICC components were simultaneously found in the $\mathrm{CHC}$ samples by pathological examination. There was no significant difference between the general characteristics of HCC patients and those of $\mathrm{CHC}$ patients, including gender and age. Fresh tissues were frozen in liquid nitrogen and used for protein extraction.

\section{Statistical analysis}

Data were presented as means \pm SD. Statistical significance between the two groups was determined using the Student's t-test. One-way ANOVA followed by the Tukey-Kramer adjustment was used to examine differences among multiple groups. $p<0.05$ was considered to be significant. All statistical analyses were conducted using SPSS 11.0.

\section{Results}

\section{Culture supernatant from intrahepatic cholangiocarcinoma cells induces EMT in hepatocellular carcinoma cells}

To examine whether ICC cells affect the behavior of HCC cells, we performed clonal formation assays using HepG2 HCC cells incubated with the culture supernatant of HCCC-9810 ICC cells. Intriguingly, the HepG2 cells grown in HCCC-9810 culture supernatant showed expansive and discrete morphology (Fig. 1A and 1B). These clones were distinctly different from the HepG2 clones cultured in the supernatant of HepG2 cells: they did not form solid (island-like) colonies (Fig. 1A) and the single-cell had a fusiform appearance, which was different from the polygon shape of HepG2 cells (Fig. 1B). Similar cellular morphology was also observed in PLC/PRF/5 HCC cells after treated with the culture supernatant of HCCC-9810 cells (Fig. 1A and 1B).

To confirm these observations were not unique to HCC cells in HCCC-9810 ICC cell culture supernatant, similar studies were performed using other ICC cells and HCC cells. When HepG2 cells were treated with the culture supernatant of HuCCT1 cells, similar cell morphology was observed (Fig. 1C). SNU-449 cells, an invasive HCC cell line, tend to form loose clones compared to HepG2 cells. Similarly, SNU-449 cells grew more sparsely when cultured in the supernatant of HuCCT1 cells (Fig. 1C).
The lack of clear apical and lateral membranes and loose or no interactions among cells are the morphological characteristics of EMT [8]. Based on the observed morphological changes described above, the molecular markers for EMT were examined. The epithelial marker E-cadherin was found to be downregulated in HepG2 cells incubated with culture supernatant from ICC cells compared with that incubated with culture supernatant from HCC cells (Fig. 1D). Conversely, mesenchymal-associated proteins Vimentin and Snail were upregulated in HepG2 and SNU-449 cells treated with culture supernatants from ICC cells (Fig. 1D). These results indicate that ICC cells create a microenvironment that induces EMT of HCC cells.

\section{Culture supernatant of intrahepatic cholangiocarcinoma cells promotes the migration and invasion of hepatocellular carcinoma cells}

The key biological significance of EMT for cancer cells is acquiring migration ability and facilitating cancer cell invasion. We next evaluated migration and invasion of HCC cells with or without adding culture supernatant of ICC cells. In wound healing assays, the culture supernatant of HuCCT1 cells induced rapid wound healing in HCC cells compared with the culture supernatant of HCC cells (HepG2 and SNU-449) (Fig. 2A). Furthermore, cells with a fusiform appearance showed increased mobility in the wound area (Fig. 2A). The results from the transwell assay also showed that culture supernatant from HuCCT1 cells enhanced the invasion ability of HepG2 and SNU-449 cells (Fig. 2B).

\section{Culture supernatant of intrahepatic cholangiocarcinoma cells does not accelerate the proliferation of hepatocellular carcinoma cells}

We next studied whether culture supernatant from ICC cells would accelerate cell proliferation of HCC cells. The results showed no significant difference between the growth rates of HCC cells in culture supernatant from HuCCT1 cells and in that from HCC cells (Fig. 3A). Results from EdU-labeling assays also showed that the culture supernatant from HCCC-9810 cells did not accelerate the proliferation of HepG2 cells (Fig. 3B).

\section{Culture supernatant of intrahepatic cholangiocarcinoma cells enhances the chemoresistance of hepatocellular carcinoma cells}

Chemoresistance is a critical obstacle to the successful treatment of cancers. We next studied the 
response of HCC cells cultured in ICC cell culture supernatant to chemo drugs. Results from drug sensitivity assays showed that HepG2 cells incubated with culture supernatant from HuCCT1 cells became more resistant to cisplatin and doxorubicin treatments compared to the cells incubated with culture supernatant from HepG2 cells (Fig. 4A). A similar result was also observed in SNU-449 cells treated with culture supernatant from HuCCT1 cells (Fig. 4A).

Apoptotic cell death was detected in these HCC cells. Results from the Annexin V staining assay
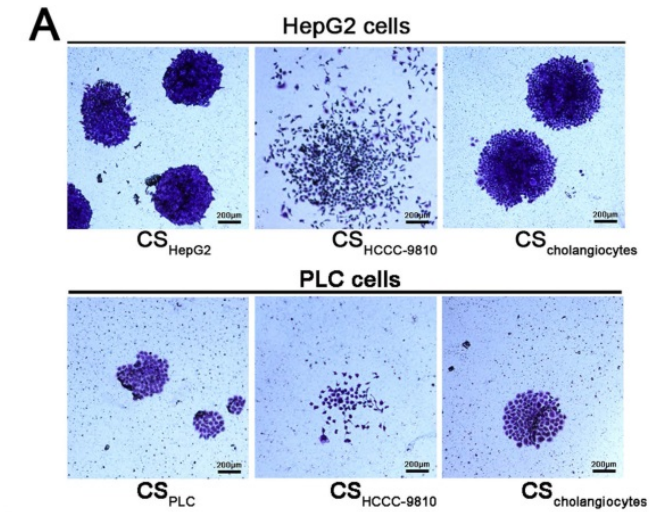

C

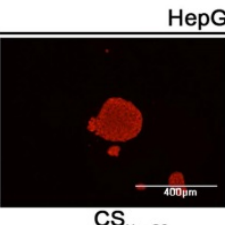

epG2 cells

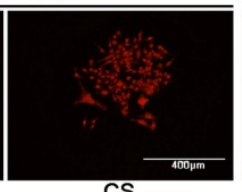

$\mathrm{CS}_{\text {Hucct1 }}$

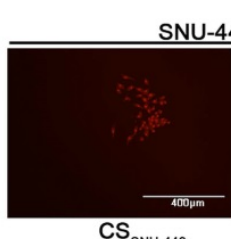

SNU-449 cells

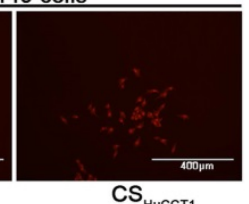

B
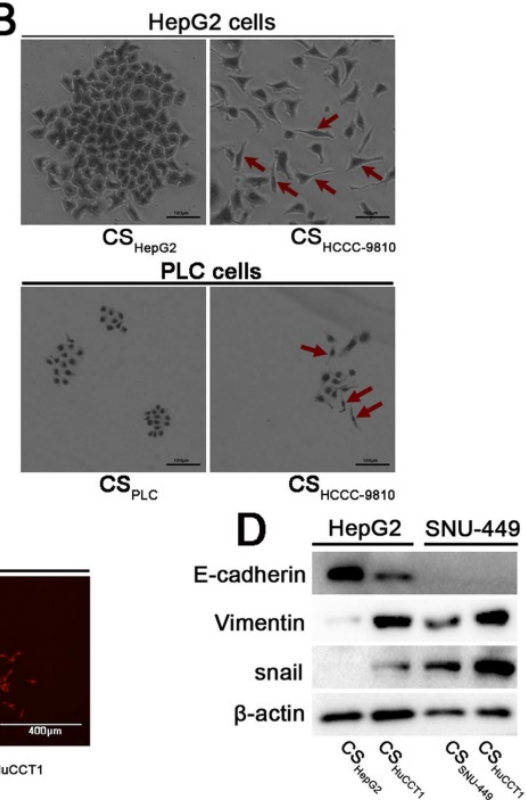

Figure 1. Culture supernatant from ICC cells induces EMT in HCC cells. (A) Colony formation assays of HepG2 and PLC/PRF/5 cells incubated with different culture supernatants. Cells were stained with crystal violet and observed by light microscopy (scale bars: $200 \mu \mathrm{m}$ ). (B) The morphology of HepG 2 cells incubated with different culture supernatants (scale bars: $100 \mu \mathrm{m}$ ). Red arrow: HepG2 cells with a fusiform shape. (C) Colony formation assays of HepG2 and SNU-449 cells incubated with culture supernatants from HuCCTl or HCC cells. These cells were stained with crystal violet and observed using fluorescence microscopy (scale bars: $400 \mu \mathrm{m})$. (D) Detection of the expression levels of E-cadherin, Vimentin, and Snail in HCC cells incubated with different culture supernatants. CS: culture supernatants; PLC cells: PLC/PRF/5 cells.
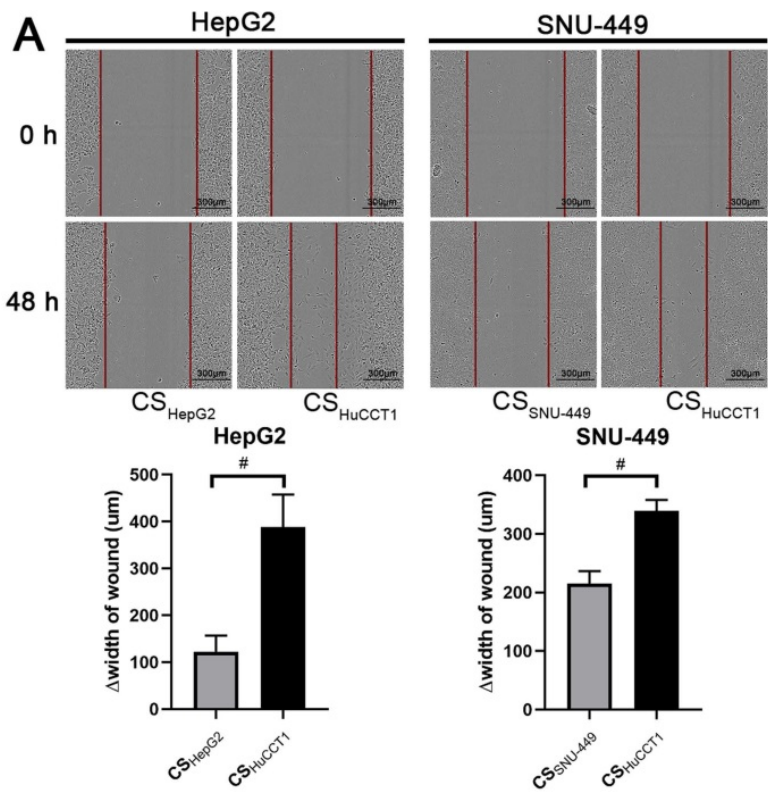

B
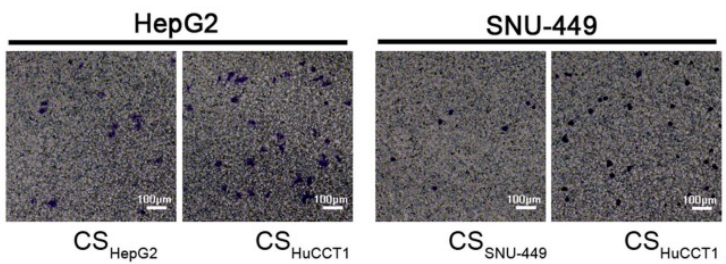

Figure 2. Culture supernatant from ICC cells promotes the migration and invasion of HCC cells. (A) Wound healing (scale bars: $300 \mu \mathrm{m})$ and (B) Transwell invasion assays (scale bars: $100 \mu \mathrm{m}$ ) of $\mathrm{HCC}$ cells incubated with different culture supernatants. $\triangle$ width of wound=width of wound at the start ( $0 \mathrm{~h}$ ) - width of wound at the end (48 h). CS: culture supernatants. \#P $<0.05$, significant differences between the two groups. 

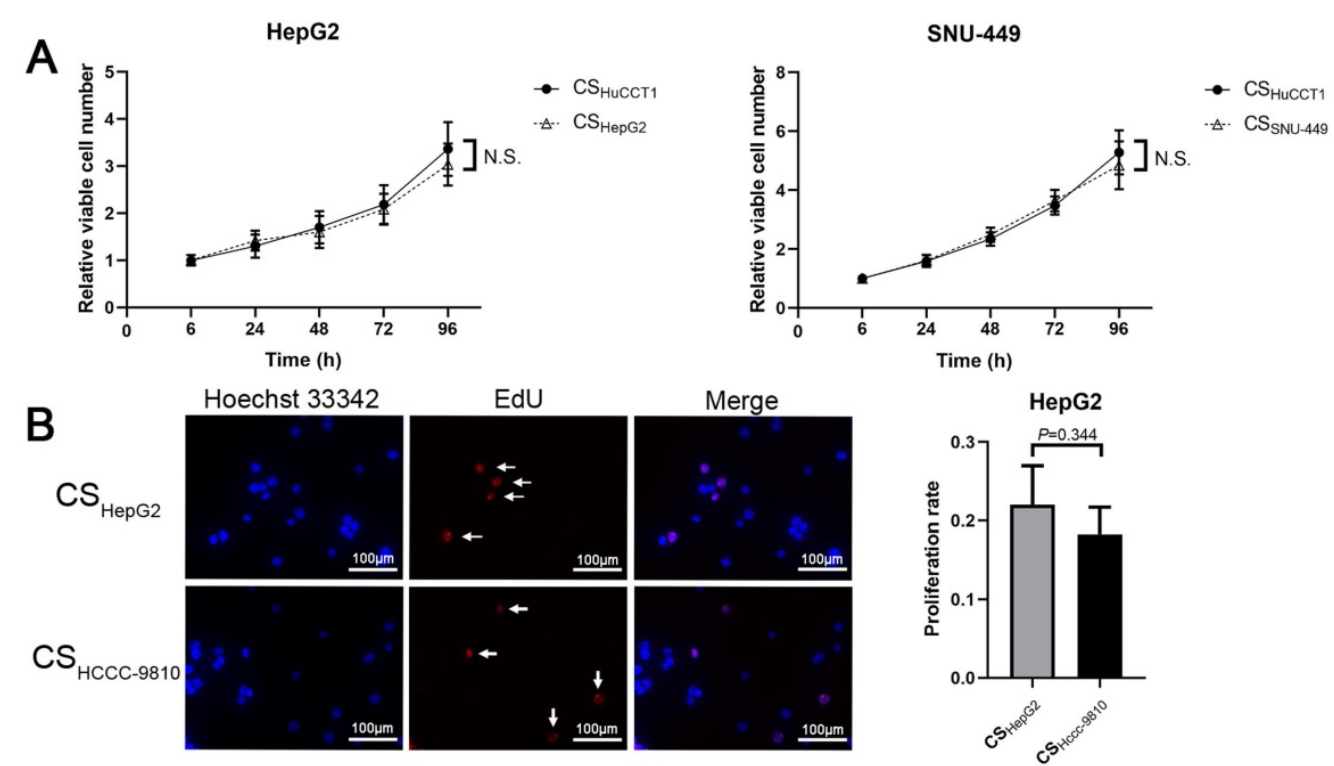

Figure 3. Culture supernatant from ICC cells does not accelerate the proliferation of HCC cells. (A) Cell proliferation and (B) EdU assays of HCC cells incubated with different culture supernatants (scale bars: $100 \mu \mathrm{m}$ ). CS: culture supernatants. N.S. = non-significant. White arrow: EdU-positive cells.
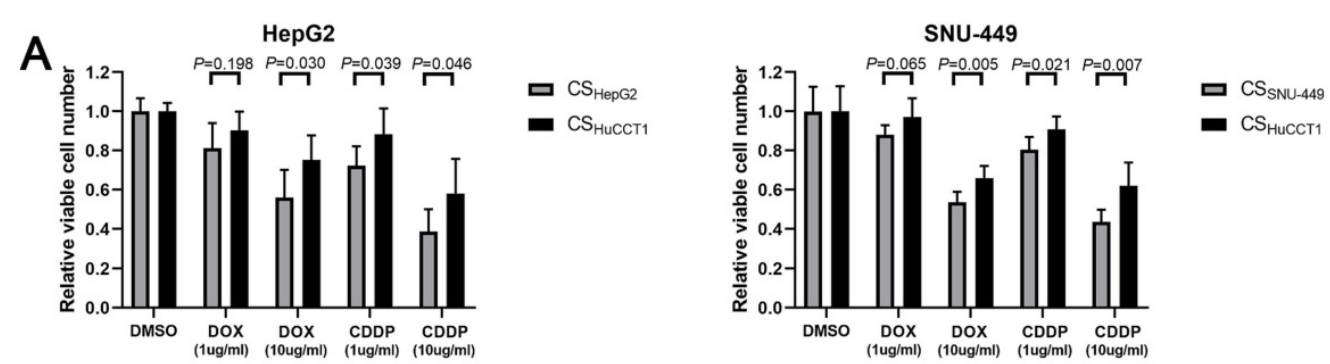

B

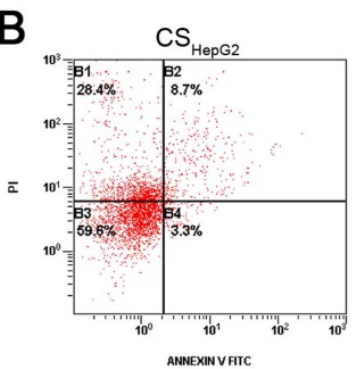

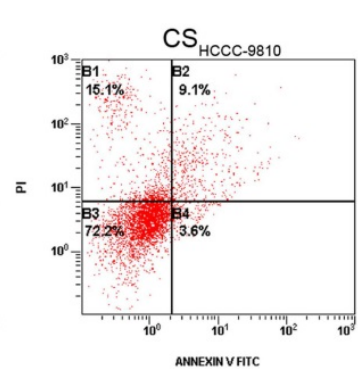
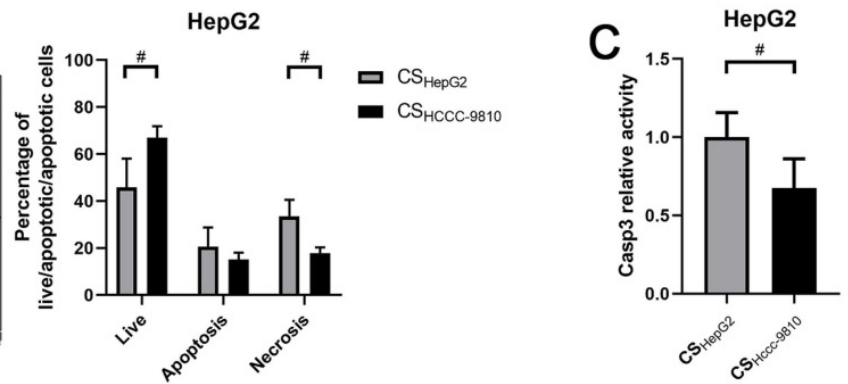

Figure 4. Culture supernatant from ICC cells enhances the chemoresistance of HCC cells. (A) Comparison of chemosensitivity of HCC cells incubated with different culture supernatants. (B) Detection of apoptosis using flow cytometry in HepG2 cells incubated with different culture supernatants and cisplatin. (C) Detection of caspase 3 activity in HepG2 cells incubated with different culture supernatants and cisplatin. CS: culture supernatants. \#P<0.05, significant differences between the two groups. CDDP: cisplatin. DOX: doxorubicin.

\section{Intrahepatic cholangiocarcinoma cells promote EMT of hepatocellular carcinoma cells through secreting LAMC2}

The tumor microenvironment plays a critical role in influencing the biological behavior of cancer cells, including EMT. A previous study reported that HCC cells showed a similar morphological change when treated with laminin-332 [18]. Given that LAMC2 encodes the most important chain of laminin-332 and it has been identified as a poor prognostic marker in some cancers $[20,21]$, the protein expression levels of LAMC2 in different ICC and HCC cells were evaluated using Western blot analysis. As shown in
Fig. 5a, LAMC2 was barely detected in HCC cell lines including HepG2 and SNU-449, but it was highly expressed in ICC HCCC-9810 and HuCCT1 cells (Fig. $5 \mathrm{~A})$.

To determine whether LAMC2 is secreted into the medium, we performed Western blot analysis using culture supernatants. LAMC2 was detected in the culture supernatant of HuCCT1 cells, while undetectable in the samples of HCC cell lines (Fig. 5B). As a control, a fresh culture medium with $10 \%$ FBS was also analyzed and LAMC2 was undetectable in these samples (Fig. 5C). These results indicate that LAMC2 is secreted by ICC cells into the culture supernatant. 


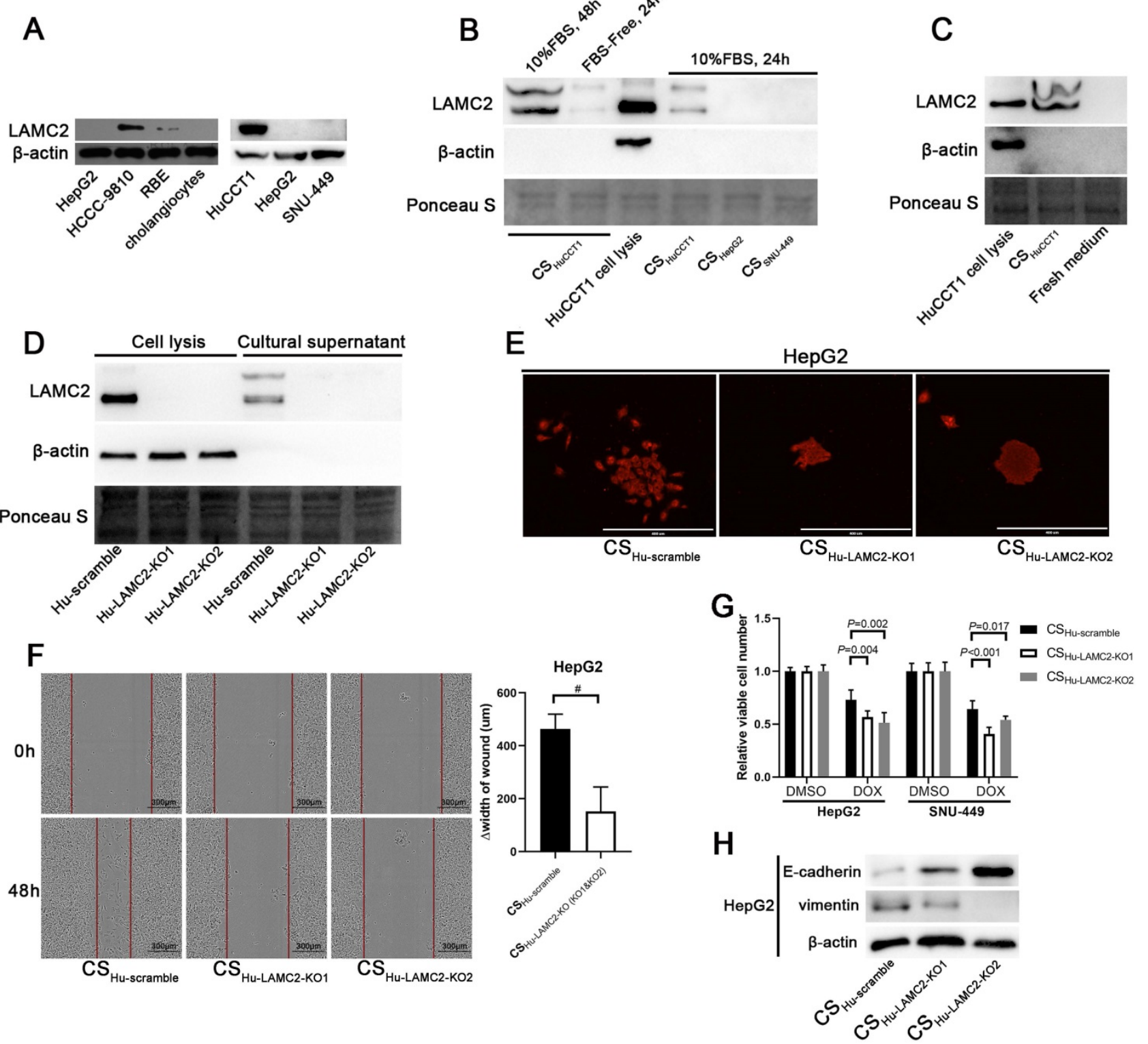

Figure 5. ICC cells induce EMT of HCC cells through secreting LAMC2. (A) Detection of LAMC2 in different ICC and HCC cells. (B) Detection of LAMC2 in different culture supernatants. The samples were loaded in the same volume $(30 \mu l)$ and ponceau $S$ was used as the loading control. The cell lysate of HuCCTI was used as a positive control. $\beta$-actin was used to detect cell lysis. Detection of LAMC2 in the fresh medium (C) and LAMC2 knockout HuCCT1 cells (D). (E) Colony formation assays of HepG2 cells incubated with culture supernatants from LAMC2 knockout HuCCT1 cells (scale bars: $400 \mu \mathrm{m}$ ). (F) Wound healing assays (scale bars: $300 \mu \mathrm{m}$ ) of HepG2 cells incubated with culture supernatants from LAMC2 knockout HuCCT1 cells. $\# P<0.05$, compared to culture supernatants from scramble clone. (G) Chemosensitivity of HCC cells incubated with culture supernatants from LAMC2 knockout HuCCT1 cells. (H) Detection of the expression levels of E-cadherin and Vimentin in HCC cells incubated with culture supernatants from LAMC2 knockout HuCCT1 cells. CS: culture supernatants.

To evaluate whether ICC cells promote EMT of ICC cells depending on LAMC2, LAMC2 KO HuCCT1 cells were established and LAMC2 was undetectable in culture supernatant from these $\mathrm{KO}$ cells (Fig. 5D). Culture supernatant from LAMC2 KO HuCCT1 cells was then used to treat HCC cells. Results from the colony formation assay showed solid colonies in HepG2 cells incubated with culture supernatant from LAMC2 KO HuCCT1 cells, different from cells incubated with culture supernatant from scramble control cells (Fig. 5E). Moreover, results from the wound healing assay in HepG2 cells also showed slowing migration when cells were incubated with culture supernatant from LAMC2 KO HuCCT1 cells (Fig. 5F). Results from drug sensitivity assays showed that HepG2 and SNU-449 cells incubated with culture supernatant from scramble HuCCT1 cells were more resistant to doxorubicin treatment compared to the cells incubated with that from LAMC2 KO HuCCT1 cells (Fig. 5G). We further observed that higher E-cadherin and lower Vimentin expression in these $\mathrm{KO}$ samples (Fig. 5H). These results indicate that LAMC2 mediates at least partially, the activity of the microenvironment of ICC cells in promoting EMT of HCC cells. 

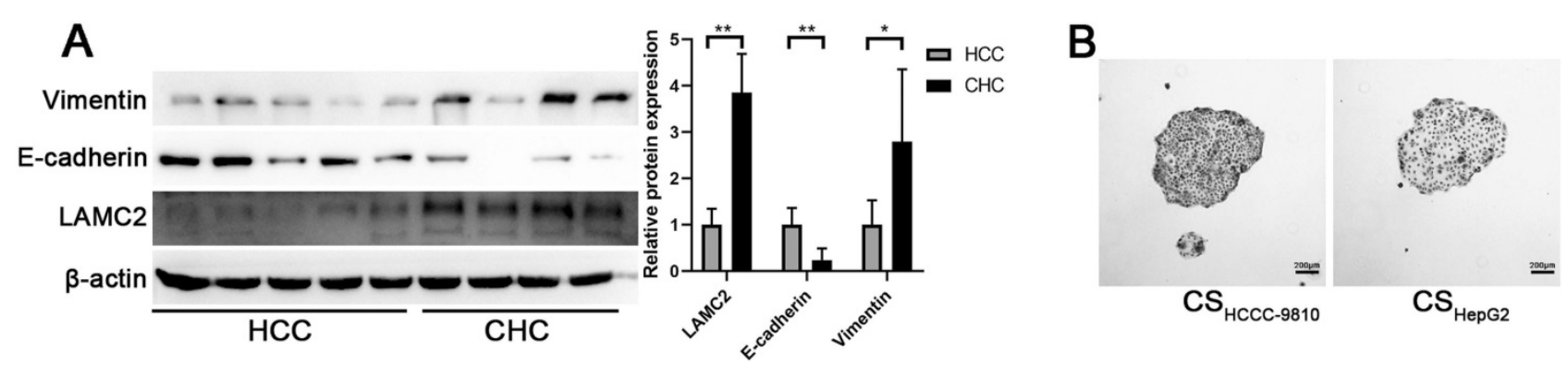

Figure 6. (A) Detection of the expression levels of LAMC2, E-cadherin, and Vimentin in human HCC and CHC tissue samples. \#P<0.05; \#P<0.01, significant differences between the two groups. (B) Colony formation assays of HCCC-9810 cells incubated with different culture supernatants (scale bars: $200 \mu \mathrm{m}$ ).

\section{High expressed LAMC2 is associated with mesenchymal phenotype in $\mathrm{CHC}$ tissues}

The expression levels of LAMC2 between $\mathrm{CHC}$ and HCC tissue samples were compared. Results from Western blot analysis showed that LAMC2 protein levels were significantly higher in $\mathrm{CHC}$ samples than those in HCC samples (Fig. 6A). Higher Vimentin and lower E-cadherin expression levels were also found in CHC samples compared to HCC samples. These results demonstrate that the levels of LAMC2 expression are positively associated with the mesenchymal phenotype in clinical CHC samples.

\section{Discussion}

CHC is defined as mixed hepatocellular carcinoma-cholangiocarcinoma, hybrid hepatocellular carcinoma-cholangiocarcinoma, or combined liver and bile duct carcinoma [4, 22]. Although it has been speculated that $\mathrm{CHC}$ may arise from hepatic progenitor cells, there is no solid evidence demonstrating the common origin of different components of $\mathrm{CHC}[23,24]$. CHC contains components of both HCC and ICC, therefore, $\mathrm{CHC}$ shows characteristics similar to HCC, such as viral hepatitis B infection and elevated alpha fetal protein (AFP), as well as those similar to ICC, such as elevated carbohydrate antigen 19-9 and lymphatic metastasis [25]. Notably, although CHC is a rare type of tumor, it has a worse prognosis and more aggressive behavior compared to HCC and ICC $[1,4,26,27]$. Given the cellular components of $\mathrm{CHC}$, we speculated that there might be interactions between the HCC and ICC components, which contribute to the poor prognosis of CHC. To study this possibility, we used culture supernatant from ICC cells to treat HCC cells, and that from HCC cells to treat ICC cells. As shown in the results (Fig. 1), morphological features of EMT are observed in HCC cells incubated with culture supernatant from ICC cells. No remarkable morphological change is observed in ICC cells incubated with culture supernatant from HCC cells (Fig. 6b). Hence, we have only focused on the effects of culture supernatant from ICC cells on HCC cells in our studies. Cancer cells with the mesenchymal phenotype show increased invasion and metastasis abilities and are associated with poor prognosis. The dismal prognosis of $\mathrm{CHC}$ is at least partially attributed to increased lymph node invasion [28]. Our results from the wound healing and invasion assays demonstrate that the migration and invasion abilities of HCC cells are enhanced after incubated with culture supernatant from ICC cells. EMT of HCC cells induced by culture supernatant from ICC cells provides a possible mechanism for the aggressive behavior of CHC. Besides enhanced metastasis, EMT is also regarded as an important contributing factor to chemoresistance $[29,30]$. Our results show that culture supernatant from ICC cells could enhance the chemoresistance of HCC cells. Together these findings provide a new rationale for the poor prognosis of $\mathrm{CHC}$.

LAMC2 encodes the $\gamma 2$ chain of laminin-332 and it is an important EMT-associated gene in various human cancers. For example, LAMC2 enhances the metastatic potential of lung adenocarcinoma through inducing EMT [15]. Upregulated LAMC2 expression in prostate cancer cells induces EMT with enhanced migration [16]. In cholangiocarcinoma, aberrant expression of LAMC2 correlates with invasion, metastasis, angiogenesis, and poor prognosis, which are attributed to EMT [31-33]. A recent study has reported that laminin-332 sustains chemoresistance and supports cell 'stemness' in hepatocellular carcinoma [34]. Studies from another report have demonstrated that laminin-332 induces EMT in hepatocellular carcinoma in vitro [18]. Our results showed that the expression level of LAMC2 is higher in ICC cells but undetectable in HCC cells. After examining the level of LAMC2 in different culture supernatants, our studies have shown that LAMC2 is present in the culture supernatants of ICC cells and accumulates over a longer period (Fig. 5B, $24 \mathrm{~h}$ vs. 48 h). Furthermore, using a LAMC2 KO cell model, our studies have demonstrated that enhanced EMT of HCC cells induced by culture supernatants from ICC 
cells is attributed to LAMC2 secreted by those ICC cells. Both CHC and ICC show worse prognoses compared to HCC $[1,35,36]$, and the prognosis of CHC is poorer than ICC [1]. A previous study has pointed out that HCC will show aggressive phenotype with shorter recurrence-free and overall survival, once cholangiocarcinoma-like genes are expressed in these HCC cells [36]. Consistent with that study [34], our results suggest that LAMC2 might be a cholangiocarcinoma-like gene causing a poor prognosis in HCC. Furthermore, our results have demonstrated that highly expressed LAMC2 is associated with increased mesenchymal phenotype in clinical CHC samples. These results suggest that promoting EMT of HCC cells by nearby ICC cells may contribute to the worse prognosis of $\mathrm{CHC}$ cases.

Collectively, our studies have demonstrated that cholangiocarcinoma cells, via the secretion of LAMC2, promote EMT of hepatocellular carcinoma cells, which may contribute to the poor prognosis of $\mathrm{CHC}$. These findings provide new insight into the interaction of different types of liver cancer cells, which may help us find a new and effective therapy for $\mathrm{CHC}$.

\section{Abbreviations}

CHC: Combined hepatocellular cholangiocarcinoma; FBS: fetal bovine serum; LAMC2: Laminin subunit gamma 2; EMT: Epithelial-mesenchymal transition; ICC: Intrahepatic cholangiocarcinoma; HCC: Hepatocellular carcinoma.

\section{Acknowledgements}

\section{Funding}

The work was sponsored by Zhejiang Provincial Natural Science Foundation of China under Grant No. LY19H160016 and LQ14H160001, National Natural Science Foundation of China (NSFC) No. 81602044, Zhejiang Provincial Public Welfare Technology Application Research Projects under Grant No. LGF18H030008, and Research Foundation of Health Bureau of Zhejiang Province under Grant No. 2020RC127, 2019ZD057 and 2018KY836.

\section{Availability of Data and Material}

The data that support the findings of this study are available from the corresponding author upon reasonable request.

\section{Ethics Committee Approval and Patient Consent}

All experimental protocols were approved by the Ethics Committee of Shaoxing People's Hospital (Shaoxing, China). All patients provided written informed consent, and Shaoxing People's Hospital
Institutional Review Board approved the tissue acquisition protocol.

\section{Author Contributions}

All authors contributed to the study's conception and design. Material preparation, data collection, and analysis were performed by Wenda Cen, Jiandong Li, Chenhao Tong, Weiguang Zhang, and Jianhua Yu. The first draft of the manuscript was written by Wenda Cen and Jianhua Yu. The first draft of the manuscript was revised by Yunfeng Zhao. The funding for this study was acquired by Weiguang Zhang, Jianhua Yu and Baochun Lu. All authors read and approved the final manuscript.

\section{Competing Interests}

The authors have declared that no competing interest exists.

\section{References}

1. Lee JH, Chung GE, Yu SJ, Hwang SY, Kim JS, Kim HY, et al. Long-term prognosis of combined hepatocellular and cholangiocarcinoma after curative resection comparison with hepatocellular carcinoma and cholangiocarcinoma. J Clin Gastroenterol. 2011; 45: 69-75.

2. Jarnagin WR, Weber S, Tickoo SK, Koea JB, Obiekwe S, Fong Y, et al. Combined hepatocellular and cholangiocarcinoma: demographic, clinical, and prognostic factors. Cancer. 2002; 94: 2040-6.

3. Liver Cancer Study Group of J. Primary liver cancer in Japan. Clinicopathologic features and results of surgical treatment. Ann Surg. 1990; 211: 277-87.

4. Gera S, Ettel M, Acosta-Gonzalez G, Xu R. Clinical features, histology, and histogenesis of combined hepatocellular-cholangiocarcinoma. World J Hepatol. 2017; 9: 300-9.

5. Yeh MM. Pathology of combined hepatocellular-cholangiocarcinoma. J Gastroenterol Hepatol. 2010; 25: 1485-92.

6. Koh KC, Lee H, Choi MS, Lee JH, Paik SW, Yoo BC, et al. Clinicopathologic features and prognosis of combined hepatocellular cholangiocarcinoma. Am J Surg. 2005; 189: 120-5.

7. Yeung KT, Yang J. Epithelial-mesenchymal transition in tumor metastasis. Mol Oncol. 2017; 11: 28-39.

8. Larue L, Bellacosa A. Epithelial-mesenchymal transition in development and cancer: role of phosphatidylinositol 3' kinase/AKT pathways. Oncogene. 2005; 24: 7443-54

9. Qian Y, Yao W, Yang T, Yang Y, Liu Y, Shen Q, et al. aPKC-iota/P-Sp1/Snail signaling induces epithelial-mesenchymal transition and immunosuppression in cholangiocarcinoma. Hepatology. 2017; 66: 1165-82.

10. Xiao S, Chang RM, Yang MY, Lei X, Liu X, Gao WB, et al. Actin-like 6A predicts poor prognosis of hepatocellular carcinoma and promotes metastasis and epithelial-mesenchymal transition. Hepatology. 2016; 63: 1256-71.

11. Giannelli G, Fransvea E, Bergamini C, Marinosci F, Antonaci S. Laminin-5 chains are expressed differentially in metastatic and nonmetastatic hepatocellular carcinoma. Clin Cancer Res. 2003; 9: 3684-91.

12. Baba Y, Iyama KI, Hirashima K, Nagai Y, Yoshida N, Hayashi N, et al. Laminin-332 promotes the invasion of oesophageal squamous cell carcinoma via PI3K activation. Br J Cancer. 2008; 98: 974-80.

13. Berrondo C, Flax J, Kucherov V, Siebert A, Osinski T, Rosenberg A, et al. Expression of the Long Non-Coding RNA HOTAIR Correlates with Disease Progression in Bladder Cancer and Is Contained in Bladder Cancer Patient Urinary Exosomes. PLoS One. 2016; 11: e0147236.

14. Kevans D, Wang LM, Sheahan K, Hyland J, O'Donoghue D, Mulcahy H, et al. Epithelial-mesenchymal transition (EMT) protein expression in a cohort of stage II colorectal cancer patients with characterized tumor budding and mismatch repair protein status. Int J Surg Pathol. 2011; 19: 751-60.

15. Moon YW, Rao G, Kim JJ, Shim HS, Park KS, An SS, et al. LAMC2 enhances the metastatic potential of lung adenocarcinoma. Cell Death Differ. 2015; 22: 1341-52.

16. Drake JM, Barnes JM, Madsen JM, Domann FE, Stipp CS, Henry MD. ZEB1 coordinately regulates laminin-332 and \{beta\} 4 integrin expression altering the invasive phenotype of prostate cancer cells. J Biol Chem. 2010; 285: 33940-8.

17. Wang H, Cai J, Du S, Wei W, Shen X. LAMC2 modulates the acidity of microenvironments to promote invasion and migration of pancreatic cancer cells via regulating AKT-dependent NHE1 activity. Exp Cell Res. 2020; 391: 111984. 
18. Giannelli G, Bergamini C, Fransvea E, Sgarra C, Antonaci S. Laminin-5 with transforming growth factor-beta1 induces epithelial to mesenchymal transition in hepatocellular carcinoma. Gastroenterology. 2005; 129: 1375-83.

19. Yu J, Zhang W, Zhang R, Jiang G, Tang H, Ruan X, et al. Molecular hydrogen attenuates hypoxia/reoxygenation injury of intrahepatic cholangiocytes by activating Nrf2 expression. Toxicol Lett. 2015; 238: 11-9.

20. Korbakis D, Dimitromanolakis A, Prassas I, Davis GJ, Barber E, Reckamp KL, et al. Serum LAMC2 enhances the prognostic value of a multi-parametric panel in non-small cell lung cancer. Br J Cancer. 2015; 113: 484-91.

21. Xue LY, Zou SM, Zheng S, Liu XY, Wen P, Yuan YL, et al. Expressions of the gamma2 chain of laminin- 5 and secreted protein acidic and rich in cysteine in esophageal squamous cell carcinoma and their relation to prognosis. Chin J Cancer. 2011; 30: 69-78.

22. Allen RA, Lisa JR. Combined liver cell and bile duct carcinoma. Am J Pathol. 1949; 25: 647-55.

23. Wang AQ, Zheng YC, Du J, Zhu CP, Huang HC, Wang SS, et al. Combined hepatocellular cholangiocarcinoma: Controversies to be addressed. World J Gastroenterol. 2016; 22: 4459-65.

24. Tanaka S, Yamamoto T, Tanaka H, Kodai S, Ogawa M, Ichikawa T, et al. Potentiality of combined hepatocellular and intrahepatic cholangiocellular carcinoma originating from a hepatic precursor cell: Immunohistochemical evidence. Hepatol Res. 2005; 32: 52-7.

25. Libbrecht L. Hepatic progenitor cells in human liver tumor development. World J Gastroenterol. 2006; 12: 6261-5.

26. Zuo HQ, Yan LN, Zeng Y, Yang JY, Luo HZ, Liu JW, et al. Clinicopathological characteristics of 15 patients with combined hepatocellular carcinoma and cholangiocarcinoma. Hepatobiliary Pancreat Dis Int. 2007; 6: 161-5.

27. Maximin S, Ganeshan DM, Shanbhogue AK, Dighe MK, Yeh MM, Kolokythas $\mathrm{O}$, et al. Current update on combined hepatocellular-cholangiocarcinoma. Eur J Radiol Open. 2014; 1: 40-8.

28. Okuda K. Natural history of hepatocellular carcinoma including fibrolamellar and hepato-cholangiocarcinoma variants. J Gastroenterol Hepatol. 2002; 17: 401-5.

29. Fischer KR, Durrans A, Lee $\mathrm{S}$, Sheng J, Li F, Wong ST, et al. Epithelial-to-mesenchymal transition is not required for lung metastasis but contributes to chemoresistance. Nature. 2015; 527: 472-6.

30. Brozovic A. The relationship between platinum drug resistance and epithelial-mesenchymal transition. Arch Toxicol. 2017; 91: 605-19.

31. Pei YF, Liu J, Cheng J, Wu WD, Liu XQ. Silencing of LAMC2 Reverses Epithelial-Mesenchymal Transition and Inhibits Angiogenesis in Cholangiocarcinoma via Inactivation of the Epidermal Growth Factor Receptor Signaling Pathway. Am J Pathol. 2019; 189: 1637-53.

32. Liu W, Tian F, Jiang P, Zhao X, Guo F, Li X, et al. Aberrant expression of laminin gamma2 correlates with poor prognosis and promotes invasion in extrahepatic cholangiocarcinoma. J Surg Res. 2014; 186: 150-6.

33. Aishima S, Matsuura S, Terashi T, Taguchi K, Shimada M, Maehara Y, et al. Aberrant expression of laminin gamma 2 chain and its prognostic significance in intrahepatic cholangiocarcinoma according to growth morphology. Mod Pathol. 2004; 17: 938-45.

34. Govaere O, Wouters J, Petz M, Vandewynckel YP, Van den Eynde K, Van den Broeck A, et al. Laminin-332 sustains chemoresistance and quiescence as part of the human hepatic cancer stem cell niche. J Hepatol. 2016; 64: 609-17.

35. Blechacz B, Gores GJ. Cholangiocarcinoma: advances in pathogenesis, diagnosis, and treatment. Hepatology. 2008; 48: 308-21.

36. Woo HG, Lee JH, Yoon JH, Kim CY, Lee HS, Jang JJ, et al. Identification of a cholangiocarcinoma-like gene expression trait in hepatocellular carcinoma. Cancer Res. 2010; 70: 3034-41. 